\title{
Evaluación eco-fisiológica de procedencias de Schizolobium parahyba subesp. amazonicum en la etapa de muda y su crecimiento en el campo
}

\author{
Eco-physiological evaluation of provenances of Schizolobium parahyba subsp. amazonicum in the
} moltingstage and its growth in the field

Avaliação eco-fisiológica das procedências de Schizolobium parahyba subsp. amazonicum na fase de muda e seu crescimento no campo

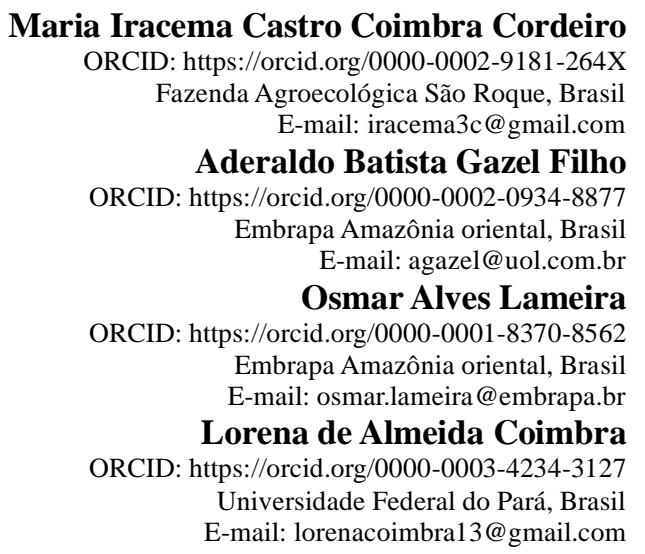

\begin{abstract}
Resumen
Las procedencias de las semillas son importantes para el establecimiento de plantaciones forestales. El objetivo del estudio fue evaluar la subespiación en caracteres eco-fisiológicos de procedencias de $S$. parahyba subesp. amazonicum (Huber ex Ducke) Barneby en la etapa de vivero y su crecimiento inicial en campo. En la etapa de vivero se evaluó la tasa de fotosíntesis, transpiración, conductancia estomática, altura y diámetro del tallo, y distribución de materia seca; en la etapa de campo se evaluó la supervivencia de las plantas y su crecimiento en altura y diámetro dos años después de la siembra. Se utilizaron las procedencias Ji-Paraná (RO), Belterra (PA), Alta floresta (MT) y Brasiléia (AC). En vivero se utilizó un diseño experimental completamente al azar y en campo un diseño de bloques completos al azar con tres repeticiones. Los parámetros fotosíntesis, conductancia estomática y transpiración en la fase de vivero mostraron subespiación significativa entre procedencias $(\mathrm{p} \leq 0.05)$, donde la procedencia Brasiléia presentó tasa mayor de conductancia estomática y transpiración, mientras Alta floresta valores mayores de fotosíntesis. Las procedencias Ji-Paraná y Alta Floresta, en la fase de vivero, por presentar mayor asignación de biomasa del tronco $(56.9 \%)$ y radicial $(52.2 \%)$ respectivamente, demostró potencial mayor para el uso en silvicultura por lo que poderá propiciar mejor desempeño de las plantas en condiciones de campo.
\end{abstract}

Palabras clave: Biomasa; Condutancia estomática; Crecimiento; Fotosíntesis; Transpiración.

\begin{abstract}
Seed provenances are important for the establishment of forest plantations. The objective of the study was to evaluate subspiation in echo-physiological characters of provenances of S. parahyba subesp. amazonicum (Huber ex Ducke) Barneby in the nursery stage and its initial growth in the field. In the nursery stage, the photosynthesis rate, transpiration, stomatal conductance, stem height and diameter, and dry matter distribution were evaluated; In the field stage, the survival of the plants and their growth in height and diameter were evaluated two years after sowing. The provenances Ji-Paraná (RO), Belterra (PA), Alta floresta (MT) and Brasiléia (AC) were used. . A completely randomized experimental design was used in the nursery and a randomized complete block design with three repetitions in the field. The parameters photosynthesis, stomatal conductance and transpiration in the nursery phase showed significant subspiation between provenances $(\mathrm{p} \leq 0.05)$, where the Brasiléia provenance presented a higher rate of stomatal conductance and transpiration, while Alta forest had higher values of photosynthesis. The Ji-Paraná and Alta Floresta provenances, in the nursery phase, because they presented a higher allocation of trunk biomass $(56.9 \%)$ and root $(52.2 \%)$ respectively, showed greater potential for use in forestry so that it will be able to promote better performance of plants under field conditions.
\end{abstract}


Keywords: Native species; Biomass; Growth; Photosynthesis; Conductance; Transpiration.

\section{Resumo}

A procedência das sementes é importante para o estabelecimento de plantações florestais. O objetivo do estudo foi avaliar a subespiação em caracteres ecofisiológicos de procedências de S. parahybasubesp. amazonicum (Huber ex Ducke) Barneby em berçário e seu crescimento inicial no campo. No estágio de berçário, foram avaliadas a taxa de fotossíntese, transpiração, condutância estomática, altura e diâmetro do caule e distribuição de matéria seca; Na fase de campo, avaliou-se a sobrevivência das plantas e seu crescimento em altura e diâmetro, dois anos após a semeadura, utilizando-se as procedências Ji-Paraná (RO), Belterra (PA), Alta floresta (MT) e Brasiléia (AC). . O delineamento experimental foi inteiramente casualizado no viveiro e o delineamento em blocos casualizados com três repetições em campo. Os parâmetros fotossíntese, condutância estomática e transpiração na fase de viveiro apresentaram subespiação significativa entre procedências $(\mathrm{p} \leq 0,05)$, onde a proveniência Brasiléia apresentou maior índice de condutância estomática e transpiração, enquanto a floresta Alta apresentou maiores valores de fotossíntese. As procedências Ji-Paraná e Alta Floresta, na fase de viveiro, por apresentarem maior alocação de biomassa de tronco $(56,9 \%)$ e raiz $(52,2 \%)$ respectivamente, apresentaram maior potencial de uso na silvicultura para que possa promover melhor desempenho de plantas em condições de campo.

Palavras-chave: Espécie nativa; Biomassa; Condutância; Crescimento; Fotossíntese; Transpiração.

\section{Introducción}

El Schizolobium parahyba subesp. amazonicum (Huber ex Ducke) Barneby (gavilán) pertenece a la familia Fabaceae. Se distribuye de manera natural en la Amazonía, especialmente en Brasil, en las zonas de bosques primarios y secundarios (Ducke, 1939). Es un árbol pionero que mide 15 a $40 \mathrm{~m}$ de altura y 50 a $100 \mathrm{~cm}$ de diámetro, de crecimiento rápido, buena adaptabilidad y con grand potencial productivo (Cordeiro et al., 2015). La diversidad de uso y valor económico de la especie ha promovido la expansión de su cultivo en la recuperación de áreas degradadas, plantaciones forestales homogénea, consorcios y agroforestales (Cordeiro et al., 2016). La ABRAF (Asociación Brasileña de Productos de Bosque Plantados, 2013) reportó 159,000 ha cubiertas con plantaciones con esta especie en el estado de Pará, Maranhão, y Tocantins.

Han surgido algunas inquietudes con respecto a la subespiación encontrada en las plantaciones establecidas en la región (Cordeiro et al., 2015). Esa subespiación se atribuye al uso de semilla de subespios árboles y origen geográfico diverso, sin control (Costa et al., 2015). No obstante, la alta subespiabilidad y el desconocimiento en cuanto a la adaptación de estos genotipos a diferentes condiciones ambientales limita el establecimiento de plantíos más productivos (Ohashi et al., 2010).

La importancia del estudio con semillas originarias de diferentes localidades geográficas permite verificar diferencias fenotípicas que se determinan por las subespiaciones ambientales que reflejan alguna expresión del genotipo (Cruz et al.,2003).

Un programa de Mejoramiento Genético es de extrema importancia para el estudio de la subespiabilidad que ocurre entre las procedencias (Ohashi et al., 2010). Esa información indica cuál es la procedencia con mejores características para ser cultivada en una plantación forestal en una determinada área.

La subespiación entre procedencias ha sido estudiada ampliamente en especies forestales. Liu et al. (2002); SoteloMontes et al. (2003); Lopez-Uptonet al. (2005), Ouedraogo, et al. (2012) demuestran en distintas especies la existencia de diferencias entre procedencias en los componentes químicos, crecimiento en altura, DAP, supervivencia y producción de biomasa. El conocimiento de la subespiabilidad en caracteres de valor económico y adaptativo en una determinada especie es de importancia en la selección de materiales más adaptados, resistentes a factores adversos en el área de reforestación y más productivos, para mejorar el desempeño y beneficio económico y ecológico de las plantaciones (Trugilhob et al., 2015).

Estudios de subespiación entre procedencias de S. amazonicum se han encontrado diferencias significativas para subespios caracteres, tanto en la fase de vivero como en el campo (Ohashi et al., 2010), lo cual se traduce en ganancia genética con la selección de las procedencias mejores (Rocha et al.,2009). A pesar del avance en la investigación de la especie aún hay vacios de información en relación con las características eco-fisiológicas entre procedencias y su adaptación al área de cultivo. El conocimiento básico de la eco-fisiología de la producción forestal y su relación a los factores ambientales es esencial para el 
uso adecuado de material genético y asegurar el éxito de una repoblación forestal (Tatagiba et al., 2008). El estudio de procesos fisiológicos permite una mejor comprensión de los factores que influyen en el metabolismo de las plantas de diferentes orígenes geográficos (Ouedraogo et al. 2012).

La hipótesis a probar es que plantas de S. amazonicum de diferentes orígenes geográficos difieren en sus características eco-fisiológicas relacionadas con el crecimiento en altura y diámetro en individuos juveniles. El objetivo fue evaluar la subespiación en caracteres eco-fisiológicos de cuatro procedencias de $S$. parahyba subesp. amazonicum en la etapa de vivero y su crecimiento inicial en campo.

\section{Materiales y Métodos}

El estudio se desarrolló en dos etapas. La primera fue la producción de plantas en el vivero forestal del Instituto de Ciencias Agrarias situado en el Campus de la Universidad Federal Rural de la Amazonia, en Belém (PA). La segunda etapa se llevó a cabo en el campo experimental de la finca Tramontina ubicada en Aurora do Pará (PA) entre las coordenadas geográficas $02^{\circ} 08^{\prime} 02^{\prime}$ 'S e 4733'32'’O, a $210 \mathrm{Km}$ de distancia de la capital, Belém. En las dos etapas de la investigación, se utilizo una metodología de naturaleza cuantitativa basada en los postulados de Hernández-Sampieri, Fernández-Collado y Baptista-Luci (2014).

Las semillas de las procedencias del estudio Ji-Paraná (RO), Belterra (PA), Alta floresta (MT) y Brasiléia (AC), se adquirieron en el laboratorio de semillas de la Asociación de las Industrias Madereras del Estado de Pará (AIMEX). Los cuatro orígenes geográficos se localizan dentro del área de distribución natural de la especie en la Amazonia Brasileña (Cuadro 1).

Cuadro 1. Localización geográfica y características edafo-climáticas de los sitios de origen de las semillas de S. Parahyba supesp. Amazonicum.

\begin{tabular}{|c|c|c|c|c|}
\hline \multirow[b]{2}{*}{ Características } & \multicolumn{4}{|l|}{ Procedencia } \\
\hline & $\begin{array}{l}\text { Brasiléia } \\
\text { (AC) }\end{array}$ & $\begin{array}{l}\text { Belterra } \\
\text { (PA) }\end{array}$ & $\begin{array}{l}\text { JI Paraná } \\
\text { (RO) }\end{array}$ & $\begin{array}{l}\text { Alta Floresta } \\
\text { (MT) }\end{array}$ \\
\hline Altitud (m) & 250 & 152 & 170 & 283 \\
\hline Latitud S & $11^{\circ} 00^{\prime} 36^{\prime \prime}$ & $02^{\circ} 38^{\prime} 09^{\prime \prime}$ & $10^{\circ} 53^{\prime} 07^{\prime \prime}$ & $09^{\circ} 52^{\prime} 32^{\prime \prime}$ \\
\hline Longitud O & $68^{\circ} 44^{\prime} 52^{\prime \prime}$ & $54^{\circ} 56^{\prime} 13^{\prime \prime}$ & $61^{\circ} 57^{\prime} 06^{\prime \prime}$ & $56^{\circ} 05^{\prime} 10^{\prime \prime}$ \\
\hline T.máx. $\left({ }^{\circ} \mathrm{C}\right)$ & 36,5 & 31,8 & 25,1 & 38 \\
\hline T.min. $\left({ }^{\circ} \mathrm{C}\right)$ & 11,1 & 25,1 & 12 & 14,2 \\
\hline Precipitación anual (mm) & 1680 & 1909 & 2133 & 2373 \\
\hline Humedad relativa (\%) & 85 & 86,7 & 81,6 & 85 \\
\hline Tipo de Suelo & $\mathrm{AVd}^{\circ}$ & $\operatorname{LAd}^{\boldsymbol{\Lambda}}$ & $\mathrm{LVa}^{\bullet}$ & Ava" \\
\hline
\end{tabular}

${ }^{\circ}$ Arcilloso rojo distrófico; ${ }^{\wedge}$ Latosol amarillo distrófico; ${ }^{\bullet}$ Latosol rojo - amarillo; ‘ Arcilloso rojo - amarillo. Fuente: IBGE (2013).

Las semillas fueron escarificadas mecánicamente previo a la producción de las plántulas, luego se sumergieron en agua durante 24 horas y después se sembraron en bolsas de polietileno $(17 \mathrm{~cm} \mathrm{x} 28 \mathrm{~cm})$, en un sustrato con mezcla de $70 \%$ de tierra negra, $20 \%$ de estiércol de aves descompuesto y $10 \%$ de arena siguiendo los procedimientos utilizados comúnment para la especie. El mantenimiento incluyó el control de malezas e irrigación manual en los días sin lluvia en el sitio.

En vivero se utilizó un diseño experimental completamente al azar, con cuatro tratamientos (procedencias) y tres repeticiones. Las plantas cultivadas en un invernadero con luminosidad interna reducida a $75 \%$ de luz natural por una media 
sombra ubicada en la parte superior externa de la estructura, con humedad relativa alrededor de $85 \%$ y una temperatura promedio de $27^{\circ} \mathrm{C}$. Tres meses después de la siembra se midió la tasa de fotosíntesis, la tasa de transpiración y la conductancia estomática), con un medidor portátil de fotosíntesis, (IRGA, Modelo LI-3000 LI-Cor, Inc, Lincoln, EUA) con una cámara de asimilación modelo LI-3050. Las medidas se tomaronen el período matutino, entre las $8 \mathrm{~h}$ y las $11 \mathrm{~h}$, en tres plantas por tratamiento, utilizando la hoja más extendida y sana desde el ápice de la planta.

También se midieron caracteres de crecimiento, incluyendo la altura total (Alt), con una regla graduada; el diámetro del tallo (DC), con un calibrador digital; y el número de hojas (NF) por el recuento. Posteriormente, se cosecharon las plantas para evaluar la biomasa. Se obtuvo el peso fresco de cada planta, luego se la subes pon y se separó la raíz, tallo y hojas. En seguida se colocaron en estufa a $75^{\circ} \mathrm{C}$, durante 72 horas hasta peso constante para obtener la materia fresca (PMF) y seca (PMS), de la hoja (F), tallo (C), raíz (R) y total (T).

En la fase de campo se estableció un experimento con un diseño de bloques completos al azar con cuatro tratamientos (procedencias) y tres repeticiones. La unidad experimental incluyó 20 plantas. Previo a la plantación se preparó el terreno con arado simple y rastrillado. La plantación se realizó en cepas (hoyos) de 30 × 30 x $30 \mathrm{~cm}$ con un espaciamiento de 4.0 x $3.0 \mathrm{~m}$ entre plantas. Todas las plantas recibieron abono orgánico al momento de la plantación, con $600 \mathrm{~g}$ de estiércol de ave descompuesto. Durante el experimento se eliminó la maleza, mediante prácticas de cultivo y aporcado. Después del control de malezas se fertilizó con abono químico de cobertura con 170 g/planta de NPK en proporción 10:20:20 dos veces al año, al principio y al final del período lluvioso. A los 24 meses de la plantación se obtuvieron los datos de supervivencia, diámetro a la altura del pecho (DAP) y altura total (HT) de las plantas.

El análisis estadístico en la fase de vivero y en campo incluyó un análisis de subespianza y una comparación de los promedios entre las procedencias con la prueba "Student Newman Keuls" - SNK, $\alpha$ " ( $\mathrm{p} \leq=0.05$ ) usando SISSUBESP 5.8 (Ferreira, 2010), porque conserva las características lógicas de la aplicación de la prueba de Duncan, pero usa la distribución de la prueba de Tukey. El método "Keuls" es relativamente fácil de aplicar y considera el número total de tratamientos utilizados en el experimento; siendo un test de amplitud múltiple que evalúa la significancia de un conjunto de diferencias, haciendo un balance entre los Errores de Tipo I y II. Los valores de medios del porcentaje de supervivencia se transformaron a través de la función raíz cuadrada de arcoseno para cada observación y el número promedio de hoja transformadas en $\sqrt{\boldsymbol{x}+\mathbf{0 . 5}}$ (Pimentel Gomes, 2009), donde $\mathrm{x}=$ datos muestreados. También se estimó la correlación lineal de Pearson entre las subespiables conductancia estomática, tasa de fotosíntesis, y tasa de transpiración de las plantas en el vivero utilizando los promedios encada repetición.

\section{Resultados y Discusión}

\subsection{Fotosíntesis, conductancia y transpiración de plantas}

Se encontraron diferencias significativas $(\mathrm{P} \leq 0.05)$ entre las procedencias para las subespiables fotosíntesis, conductancia estomática y transpiración en la fase de vivero. Las procedencias Alta Floresta y Brasiléia presentaron mayores valores de tasa fotosintética que las procedencias Belterra y Ji-Paraná (Cuadro 2). Esto significa que Alta Floresta y Brasiléia son capaces de aprovechar mejor las condiciones de energía disponible. La menor tasa fotosintética de Ji-Paraná, indica una reducción en la asimilación de $\mathrm{CO} 2$ y su conducción en la hoja, consecuentemente reduciendo la concentración de ese elemento en los espacios intercelulares.

Okuto (2010) encontró que las procedencias de Syzygium cuminii difieren en la tasa de CO2 fijado, aunque la tasa también es afectada por la edad de la planta. La fotosíntesis es uno de los procesos que afectan la asimilación y uso de los 
recursos tales como carbono, agua y nutrientes. Estos factores influyen directamente en el crecimiento de la planta y son aspectos importantes en la selección del material genético.

Cuadro 2. Valores promedio de los caracteres fisiológicos evaluados en cuatro procedencias de Schizolobium parahyba subesp. amazonicum UFRA, en Belém, Pará, Brasil.

\begin{tabular}{|c|c|c|c|}
\hline \multirow{3}{*}{$\begin{array}{l}\text { Procedencias } \\
\text { de las simientes }\end{array}$} & \multicolumn{3}{|c|}{ Parámetros fisiológicos } \\
\hline & Fotosíntesis & Conductancia & Transpiración \\
\hline & $\left(\mu \mathrm{mol} \mathrm{CO} 2 \mathrm{~m}^{-2} \mathrm{~s}^{-1}\right)$ & \multicolumn{2}{|c|}{$\left(\mathrm{mmol} \mathrm{H} \mathrm{H}_{2} \mathrm{Om}^{-2} \mathrm{~s}^{-1}\right)$} \\
\hline Alta Floresta & $9.93 \mathrm{a}$ & $0.76 \mathrm{~b}$ & $4.40 \mathrm{~b}$ \\
\hline Brasiléia & $9.60 \mathrm{a}$ & $2.14 \mathrm{a}$ & $6.20 \mathrm{a}$ \\
\hline Belterra & $5.48 \mathrm{~b}$ & $0.63 \mathrm{~b}$ & $4.09 \mathrm{~b}$ \\
\hline Ji Paraná & $2.81 \mathrm{c}$ & $0.36 \mathrm{~b}$ & $2.95 \mathrm{c}$ \\
\hline $\mathrm{CV}(\%)$ & 37.32 & 74.38 & 24.90 \\
\hline Promedio. & 6.95 & 1.04 & 4.31 \\
\hline
\end{tabular}

Letras iguales en la misma columna, indican que no hay diferencia significativa conp $=0.05$ de probabilidad por la prueba de SNK. Elaboración: Autores.

Para la conductancia estomática y transpiración, la procedencia Brasiléia presentó una tasa significativamente mayor en relación a las demás procedencias. El menor valor de conductancia estomática de la procedencia Ji-Paraná, sugiere que la caída de la tasa de fotosíntesis esté relacionada con el cierre parcial de los estomas.

La reducción de la conductancia estomática es el principal factor limitante de la fotosíntesis (Peeva y Cornic, 2009). La conductancia estomática representa una subespiable de estado clave para predecir el uso del agua y la fotosíntesis liquida y, por lo tanto, la eficiencia del uso de la radiación y la productividad primaria (Macfarlane et al., 2004). La estimación de este parámetro es esencial en la simulación de la productividad de plantaciones y de la eficiencia del uso del agua en ecosistemas de producción (Yu et al., 2004). Gazel Filho et al. (2007) encontraron resultados semejantes en el estudio realizado entre procedencias de gavilán en estadio de plántula. Estos autores indica una fuerte dependencia de la tasa de fotosíntesis con relación a conductancia estomática, teniendo en cuenta que valores elevados de conductancia proporcionan valores elevados de fotosíntesis.

Las especies forestales son responsables por la transformación de $\mathrm{CO}_{2}$ en carbono orgánico para posteriormente almacenarlo en la vegetación y en el suelo, incorporándolo a su estructura mediante el intercambio de carbono con la atmósfera, a través del proceso fotosintético y la respiración (Andrade et al., 2014) y cualquier alteración estomatal, provoca reducción en la conductividad foliar, inhibiendo la fotosíntesis y la respiración de las plantas (Machado et al., 2002).

En estudios sobre alteraciones morfo-fisiológicas de Curatella amaericana L. Dalmolin et al. (2015) verificaron que la concentración de pigmentos fotosintéticos no difiere cuando las plantas se colocan en ambientes diferentes. Cualquier alteración estomática provoca reducción en la conductividad foliar, inhibiendo la fotosíntesis y la respiración de las plantas (Machado et al., 2002).

Los menores valores de transpiración observados en la procedencia Ji-Paraná, sugieren menor consumo de agua. Okuto y Ouma (2010) obtuvieron resultados similares en plantas de Syzygium cuminii de tres procedencias, lo cual corrobora nuestros resultados. El crecimiento de las plantas depende de la habilidad de los estomas para controlar la pérdida de agua. En estudios realizados por Franco y Lüttge (2002) hay una alta relación lineal entre la adquisición de $\mathrm{CO}_{2}$ y la tasa de 
transpiración.

El mismo patrón de respuesta se encontró en clones de Eucaliptus, los que tienen mayor potencial fotosintético también alcanzan mayor tasa de transpiración (Inoue y Ribeiro, 1988). Las procedencias Alta Floresta y Ji- Paraná, a pesar de presentar mayor proximidad geográfica, presentaron mayor diferencia en el comportamiento en relación a la tasa fotosintética, revelando la existencia de diferencias genéticas entre esas procedencias para esta subespiable.

La matriz de correlación lineal de Pearson de los promedio encada repetición indicó una correlación alta entre la conductancia estomática y la transpiración $(r=0.87 ; p<0.05)$, un valor medio $(r=0.55 ; p<0.05)$ entre fotosíntesis y transpiración y un valor bajo $(r=0.39 ; \mathrm{p}<0.05)$ entre fotosíntesis y conductancia estomática.

\subsection{Crecimiento, biomasa fresca y seca de plantas}

El análisis de subespianza para las características de crecimiento de las plántulas detectó diferencias significativas (P $\leq 0.05$ ) entre las procedencias para las subespiables estudiadas (Cuadro 3).

Cuadro 3. Altura $(\mathrm{cm})$, diámetro del tallo $(\mathrm{mm})$, número de hojas biomasa (g/planta) fresca y seca de hojas, tallo y raíz de mudas de Schizolobium parahyba subesp. amazonicum de diferentes procedencias.

\begin{tabular}{|c|c|c|c|c|c|c|c|}
\hline \multirow{2}{*}{ Subespiables } & & \multicolumn{4}{|c|}{ Procedencias } & \multirow[b]{2}{*}{ Média } & \multirow[b]{2}{*}{$\mathrm{CV}(\%)$} \\
\hline & & Alta Floresta & Brasiléia & Belterra & Ji-Paraná & & \\
\hline Altura (cm) & & $40.0 \mathrm{ab}$ & $39.66 \mathrm{~b}$ & $32.67 \mathrm{~b}$ & $47.67 \mathrm{a}$ & 40 & 10.46 \\
\hline Diámetro del t & $(\mathrm{mm})$ & $0.73 \mathrm{ab}$ & $0.77 \mathrm{~b}$ & $0.67 \mathrm{c}$ & $1.06 \mathrm{a}$ & 0.81 & 4.20 \\
\hline Número de ho & & $1.79 \mathrm{a}$ & $1.83 \mathrm{a}$ & $2.24 \mathrm{a}$ & $2.07 \mathrm{a}$ & 1.98 & 11.98 \\
\hline \multirow{3}{*}{$\begin{array}{l}\text { Materia fresca } \\
\text { (g/planta) }\end{array}$} & Hoja & $6.87 \mathrm{~b}$ & $12.80 \mathrm{~b}$ & $9.32 \mathrm{~b}$ & $22.88 \mathrm{a}$ & 12.96 & 25.45 \\
\hline & Tallo & $8.07 \mathrm{~b}$ & $9.90 \mathrm{~b}$ & $7.04 \mathrm{~b}$ & $18.29 \mathrm{a}$ & 10.82 & 31.47 \\
\hline & Raíz & $15.25 \mathrm{a}$ & $21.70 \mathrm{a}$ & $15.06 \mathrm{a}$ & $18.26 \mathrm{a}$ & 17.56 & 22.57 \\
\hline \multirow{3}{*}{$\begin{array}{l}\text { Materia seca } \\
\text { (g/planta) }\end{array}$} & Hoja & $1.76 \mathrm{~b}$ & $1.86 \mathrm{~b}$ & $2.33 \mathrm{ab}$ & $3.28 \mathrm{a}$ & 2.30 & 49.40 \\
\hline & Tallo & $1.26 \mathrm{~b}$ & $2.30 \mathrm{~b}$ & $1.25 \mathrm{~b}$ & $4.82 \mathrm{a}$ & 2.15 & 57.34 \\
\hline & Raíz & $3.29^{\mathrm{a}}$ & $2.04 \mathrm{~b}$ & $3.86 \mathrm{a}$ & $1.32 \mathrm{~b}$ & 2.63 & 24.00 \\
\hline \multicolumn{2}{|l|}{ Total } & $6.31 \mathbf{b}$ & $5.86 \mathrm{~b}$ & $7.47 \mathbf{a}$ & $8.48 \mathrm{a}$ & 7.03 & 19.80 \\
\hline
\end{tabular}

Valores seguidos de las mismas letras en la columna no difieren estadísticamente entre sí por el test SNK $(\alpha=0.05) \bullet^{\bullet}$ valores transformados por la Raíz cuadrada de $\sqrt{y+0,5}$

Elaboración: Autores.

Las plantas de la procedencia Ji-Paraná fueron superiores en altura y diámetro del tallo a las de las otras procedencias, a pesar de que no difieren entre ellas en el número de hojas. Ohashi et al. (2010) al evaluarlas mismas procedencias en el municipio de Colares (PA) obsersubespon que la procedencia Brasiléia presentó menor altura de planta a los 3 meses de edad. Costa et al. (2015) encontraron subespiación en el diámetro del tallo y número de hojas a los tres meses de edad en progenies de Tectona grandis. La subespiación en el desarrollo de las plantas de diferentes orígenes geográficos sugiere diferencias entre ellas en la adaptación a las condiciones del sitio, lo que posibilita selección en etapas futuras para la mejora genética de la especie. Las diferencias observadas en el crecimiento en altura pueden ser en parte debido a la subespiación genética entre las procedencias (Ward y Lugo, 2003).

En cuanto a la materia fresca, la Procedencia Ji-Paraná fue superior en el peso de hoja y tallo; mientras que Brasiléia en el peso de raíz. Las procedencias estudiadas no presentaron diferencias significativas en el peso de materia seca de hojas, 
sin embargo, presentan valores numéricos distintos.

Asi, la procedencia Ji-Paraná presentó mayor biomasa en el tallo que las otras. Por otro lado, Belterra y Alta Floresta presentaron mayor biomasa de raíz que las procedencias Brasiléia y Ji-Paraná. Con relación al peso de materia seca total, la procedencia Ji-Paraná fue superior a las procedencias Alta Floresta y Brasiléia e igual a la procedencia Belterra, siendo esta última también igual a las procedencias Alta Floresta y Brasiléia.

Se encontró correlación negativa de alta magnitud entre materia seca del tallo y materia seca de raíz (-0.93) y de baja magnitud entre materia seca del tallo y materia seca de hojas (-0.29). Aplicando el mismo cálculo se encontró correlación positiva de magnitud media entre materia seca del tallo y materia seca total (0.58) y materia seca de raíz con materia seca de hojas (0.44), mientras que para materia seca total $(0.31)$ se detectó correlación negativa de baja magnitud.

La asignación de biomasa en las estructuras analizadas de las cuatro procedencias se verificó que Ji-Paraná direccionó $56.9 \%$ de sus fotoasimilados para el tallo, 27.5\% para hojas y 15.6\% para raíces. Brasiléia distribuyó su biomasa en cantidades prácticamente iguales entre las partes: hojas (31.6\%), tallo (33.5\%) y raíces (34.9\%).

Alta floresta destinó mayor cantidad de fotoasimilados para raíces (52.2\%), que para las hojas (27.9\%) y para el tallo (19.9\%). La Procedencia Belterra asignó parte elevada de su biomasa para la formación de raíces (52.2\%) y también para las hojas (44.4\%), siendo que esta procedencia almacenó apenas 3.42\% de sus reservas para el tallo.

Las hojas, tallos y raíces de las procedencias de gavilán presentaron asignación de biomasa de forma diferencial, siendo que Ji-Paraná por presentar mayor distribución de biomasa en el tallo indica mayor potencial para uso en silvicultura. Mientras que Alta Floresta por tener una mayor biomasa radical podrá propiciar un mejor desempeño de las mudas en condiciones de campo, especialmente en áreas degradadas, pues hay mayor posibilidad de supervivencia de la planta en razón de la mayor facilidad de sustentación y mayor área para absorción del agua y nutrientes.

\subsection{Crecimiento en el campo}

En lo que se refiere al comportamiento del crecimiento de las plantas no hubo diferencias significativas entre las procedencias a los 24 meses de edad, sin embargo, ocurrieron diferencias numéricas para la sobrevivencia con los mejores resultados para Belterra, Alta Floresta y Ji-Paraná, y menor valor para Brasiléia (Cuadro 4).

El buen rendimiento y adaptación de las procedencias de Gavilán indicaron que las condiciones de clima y suelo de la región atienden las exigencias de la especie. Ribeiro y Oliveira (2013) establecen una franja de adecuación de gavilán a los diferentes tipos de suelo del estado de Espirito Santo (Brasil) y, el latosol amarillo que existe en Aurora do Pará (Cuadro 1) está entre ellos. 
Cuadro 4. Supervivencia (\%), altura (m) y diámetro del tallo a la altura del pecho (DAP, $\mathrm{cm}$ ) de Schizolobium parahyba subesp. amazonicum (Huber ex Ducke) Barneby a los 24 meses de edad en el municipio de Aurora do Pará, estado de Pará, Brasil.

\begin{tabular}{lccc}
\hline & \multicolumn{3}{c}{ Características } \\
\cline { 2 - 4 } Procedencias & $\begin{array}{c}\text { Sobrevivencia } \\
(\%)\end{array}$ & $\begin{array}{c}\text { Altura } \\
(\mathrm{m})\end{array}$ & $\begin{array}{c}\text { DAP } \\
(\mathrm{cm})\end{array}$ \\
\hline Brasiléia-AC & $95.12 \mathrm{a}$ & $6.27 \mathrm{a}$ & $8.12 \mathrm{a}$ \\
Belterra-PA & $98.54 \mathrm{a}$ & $6.58 \mathrm{a}$ & $8.11 \mathrm{a}$ \\
Ji-Paraná-RO & $98.00 \mathrm{a}$ & $6.14 \mathrm{a}$ & $8.01 \mathrm{a}$ \\
Alta Floresta-MT & $97.35 \mathrm{a}$ & $5.88 \mathrm{a}$ & $7.61 \mathrm{a}$ \\
\hline Promedios. & & 6.23 & 7.96 \\
\hline
\end{tabular}

Valores seguidos de las mismas letras en la columna no difieren estadísticamente entre sí por el test SNK $(\alpha=0,05)$.

Elaboración: Autores.

La diferencia de las condiciones ambientales entre la procedencia Brasiléia, principalmente por las características edafológicas y el local de implantación de ensayo (Cuadro1) pueden haber contribuido para la mayor mortalidad de los individuos en el ensayo. El seguimiento de esas procedencias en etapas posteriores será de gran importancia para inferir en relación a la adaptación de esas procedencias para el local en estudio.

Ohashi et al. (2010) obsersubespon resultados semejantes evaluando las mismas procedencias en el municipio de Colares (PA). Estos autores verificaron que la procedencia Belterra presenta mayor porcentual de sobrevivencia (93.89\%) y mayores valores de altura $(7.3 \mathrm{~m})$ y DAP $(10.34 \mathrm{~cm})$ y la procedencia Brasiléia con menor porcentual de supervivencia $(70 \%)$ y desarrollo con $5.76 \mathrm{~m}$ de altura y $10.07 \mathrm{~cm}$ de diámetro. Rocha et al. (2009) obsersubespon que para Schizolobium amazonicum la supervivencia de las plantas se diferencia entre las distintas procedencias, sin embargo, la población del estado de Rondônia, presenta mayor porcentual de supervivencia.

La subespiación entre las plantas de las procedencias estudiadas probablemente esté relacionada al hecho de que poblaciones de diferentes orígenes presentaron diferencias genéticas a pesar de que estén en condiciones ambientales similares.

El gavilán tiene importancia para la recuperación productiva y ambiental de las áreas degradadas en la región y el estudio de procedencias es un paso importante cuando se usa principalmente en plantaciones comerciales para atender la industria maderera.

Junto con los estudios de procedencia, otros factores como edad de rotación de las plantas, calidad del terreno, luminosidad, nutrición, entre otros, necesitan ser considerados sobre la producción de biomasa y las interacciones entre dichos factores no observados en este estudio.

\section{Conclusiones}

Las procedencias de S. parahyba subesp. amazonicum difieren en diámetro del tallo, altura, número de hojas y asignación de biomasa en la fase de plántula en vivero. Esta subespiabilidad es importante para la selección futura en programas de mejoramiento genético de la especie.

Alta floresta valores mayores de fotosíntesis Las procedencias Ji-Paraná y Alta Floresta, en la fase de vivero, por presentar mayor asignación de biomasa del tronco (56.9\%) y radicial (52.2\%) respectivamente, demostró potencial mayor para 
el uso en silvicultura por lo que podrá propiciar mejor desempeño de las plantas en condiciones de campo.

Para la espécie en estúdio que aún no cuentan con un proceso de mejoramiento genético establecido, los resultados eco- fisiológicos se presentan como indicadores para estudios que involucran el seguimiento de otros factores físicos, químicos y biológicos que puenden influir en el metabolismo de plantas de Schizolobium de diferentes orígenes, contribuyendo a la formación de plantaciones más productivas.

\section{Reconocimiento}

Este artículo honra a la profesora Selma Toyoko Ohashi, queien vivió y se dedicó a la ciencia y la educación en la Amazonía. Nuestro agradecimiento por la inconmensurable contribución al sector florestal.

\section{Referencias}

Abraf. (2013). Anuário estatistico ano base 2012. Associação Brasileira de Produtores de Florestas Plantadas-ABRAF, Brasília. 148p.

Andrade, H. J., Segura, M. A., Canal, D. S., Feria, M., Alvrado, J. J., Marín, L.M., Pachon, D. \& Andgómez, M. J. (2014). The carbon footprint of coffee productive chains in Tolima, Colombia. En: Oelberman, M. (Ed.). Sustainable Agroecosystems in Climat e Change Mitigation. (The Netherlands). Wageningen Academic Publishers pp: 53-66.

Cordeiro, I. M. C. C., Oliveira Jr, M. C. M., Gazel Filho, A. B., Barros, P. L. C., \& Oliveira, O. A. (2016). Crecimiento del Schizolobium parahyba subesp. amazonicum cultivado en presencia de Ananas comosus subesp. Erectifolius en Pará, Brasil. Agrociencia 50(1), 79-88.

Cordeiro, I. M. C. C., Barros, P. L. C. de Lameira, \& Gazel Filho, A. B. (2015). Avaliação de plantios de paricá (Schizolobium parahyba var. amazonicum (Huber ex Ducke) Barneby de diferentes idades e sistemas de cultivo no município de aurora do Pará- Pa (Brasil). Ciência Florestal, 25 (3), $679-687$.

Costa, R. B., Martinez, Chichorro, J. F., Bauer, M. de O., Cezana, D. P., \& Souza T. R. (2015). Desempenho de progênies no pré-melhoramento de Tectona grandis L. no Estado do Espírito Santo. Scientia Forestalis 43(105), 211-216.

Cruz, C. D., Regazzi, A. J., \& Carneiro P. C. S. (2003). Modelos volumétricos aplicados ao melhoramento genético. Viçosa: Editora UFV. 2. 585p.

Dalmolin, A. C., Thomaz S. E. de O., Almeida, B. C., \& Ortiz C. E. R. (2015). Alterações Morfofisiológicas de plantas jovens de Curatella americana L. submetidas ao sombreamento. Rev. Bras.de Biociências 13, 41- 48.

Ducke, A. (1939). As Leguminosas da Amazônia Brasileira. Serviço florestal. Ministério da Agricultura. Serviço de Publicidade Agrícola. Rio de Janeiro. 88 p.

Franco, A. C., \& Lüttge, U. (2002). Midday depression in savanna trees: coordinated adjustments in photochemical, efficiency, photorespiration, CO2 assimilation and water use efficiency. Ecologia Brasiliensis. May;131(3), 356-365.

Gazel Filho, A. B., Cordeiro, I. M. C. C., Alvarado, J. R., \& Santos Filho, B. G. (2007). Produção de Biomassa em Quatro Procedências de Paricá (Schizolobium parahyba subesp. Amazonicum Huber ex Ducke) Barneby no Estádio de Muda. Rev. Bras. De Biociências 5(2), $1047-1049$.

Hernández-Sampieri, R., Fernández-Collado, C., \& Baptista-Lucio, P. (2014). Selección de la muestra. En Metodología de la Investigación (6a ed.), 170-191.

Ibge. 2013. Instituto Brasileiro de Geografia e Estatistica - IBGE. http://www.ibge.gov.br/home/geociencias/geografia/default_div_int.shtm?c=1 elemento da articulação espacial.

Inoue, M. T., \& Ribeiro, F. A. (1988). Fotossíntese e transpiração de clones de Eucalyptus grandis x E. saligna. IPEF, Piracibaba 40, 15-20.

Liu, Z., Zhou, G., Xu, S., Wu, J., \& Yin, Y. (2002). Provenance subespiation in camptothecin concentrations of Campto the caacuminata grown in China. New Forests 24, 215-224.

Lopez-Upton, J., Donahue, J. K., Plascencia-Escalante, F. O., Ramirez-Herrera, C. (2005). Provenance variation in growth characters of four subtropical pine species planted in Mexico, New Forests 29, 1-13.

Machado, E. C., Quaggio, J. A., Lagôa, A. M. M., Ticelli, A. M., \& Furlan, P. R. (1994). Trocas gasosas e relações hídricas em laranjeiras com clorose subespiegada dos citros. Revista Brasileira de Fisiologia Vegetal 6, 53-57.

Machado, E. C., Medina, C. L., Gomes, M. M. A., \& Habermann, G. (2002). Variação sazonal da fotossíntese, condutância estomática e potencial da água na folha de laranjeira 'valência'. Scientia Agricola 59(1), 53-58.

Macfarlane, C., White, D. A., \& Adams, M. A. (2004). La respuesta aparente de retroalimentación al déficit de presión de vapor de los estomas en Eucalyptus globulus Labill cultivado en el campo y sequía. Plant, Cell \& Environment, 27, 1268-1280.

Medina, C. L., \& Machado, E. C. (1999). Trocas gasosas e relações hídricas em laranjeira 'valência' enxertada sobre limoeiro 'cravo' e trifoliata e submetida à deficiência hídrica. Bragantia. 57(1). 
Research, Society and Development, v. 10, n. 1, e12410111551, 2021

(CC BY 4.0) | ISSN 2525-3409 | DOI: http://dx.doi.org/10.33448/rsd-v10i1.11551

Ohashi, S. T., Yared, J. A. G., \& Francisco Neto, J. T. (2010). Variabilidade entre procedências de paricá Schizolobium parahyba subesp amazonicum (Huber ex Ducke) Barneby plantadas no município de Colares - Pará. Acta Amazonica 40, 81-88.

Okuto, J. L., \& Oum, G. (2010). Provenance variation of photosynthesis of seedlings of java plum (S. cuminii) in Western Kenya. Agric. Biol. J. N. Am1 (6), $1260-1263$

Ouedraogo, M., Zagre M’bi, B., Jorgensen, S. T., \& Liu, F. (2012). Effect of mounding times on yield of Bambara groundnut (Vigna subterranea (L.) Verdc.) landraces in Sahel-Burkina Faso. African Journal of Agricultural Research 7 (32), 4505-4511.

Peeva, V., \& Cornic, G. (2009). Leaf photosynthesis of Haberlea Rhodopensis before and during drought. Environmental and Experimental Botany 65, 2-3.

Pimentel-Gomes, F. (2009). Curso de estatística experimental. (15a ed.), Piracicaba: Fealq-Usp. 451 p.

Ribeiro, I. O., \& Oliveira, F. B. (2013). Zoneamento Edafoclimático de Schizolobium amazonicum Huber ex Ducke utilizando análise multicritério. Anais XVI Simpósio Brasileiro de Sensoriamento Remoto - SBSR, Foz do Iguaçu (PR), Brasil.

Rocha, R. B., Vieira, A. H., Gama, M. M. B., \& Rossi, L. M. B. (2009). Avaliação genética de procedências de bandarra (Schizolobium amazonicum) utilizando REML/BLUP (Máxima verossimilhança restrita/Melhor predição linear não viciada). Scientia. Forestalis. 37(84), 351-358.

Sotelo Montes, Vidaurre, C. H., \& Weber, J. (2003). Variation in stem-growth and branch-wood traits among provenances of Calycophyllum spruceanum Benth.from the Peruvian Amazon, New Forests. 26, 1-16.

Tatagiba, S. D., Pezzopane, J. E. M., \& Reis, E. F. (2008). Relações hídricas e trocas gasosas na seleção precoce de clones de eucalipto para ambientes com diferenciada disponibilidade de água no solo. Revista Floresta. 38(2), 387-400.

Trugilho, P. F., Goulart, S. L., Assis, C. O. C., Alves, I. C. N., Protásio, T. P., \& Napoli, A. (2015). Características de crescimento, composição química, física e estimativa de massa seca de madeira em clones e espécies de Eucalyptus jovens. Ciência Rural, Santa Maria, 45(4), 661-666.

Ward, S., \& Lugo, A. (2003). Twenty Mahogany Provenances Under Different Conditions in Puerto Rico and the U.S. Virgin Islands. Ecological Studies.159, 29-93.

Yu, G-R., Wang, Q-F., \& Zhuang, J. (2004). Modeling the water use efficiency of soy be and maize plants under environmental stresses: application of a synthetic model photosynthesis-transpiration base do stomatal behavior. Journal of Plant Physiology 161, 308-318; 